\title{
Nuevas estrategias de formación profesional para la modernización del Estado en Chile
}

\author{
Díaz P., Nelson* \\ Rivera M., Claudio** \\ Krraushaar, Lilith ${ }^{\star * *}$
}

\section{Resumen}

Desde comienzos de la década de los 90 en Chile se observa un interés creciente del Estado por modernizarse. Este proceso ha sido expresado en diferentes políticas, programas e instituciones, que en conjunto pueden articularse en políticas públicas de modernización, que han sido promovidas entre los funcionarios del Estado, relevando de este modo el papel clave que los recursos humanos juegan en el éxito del proceso. En otro esfuerzo convergente hacia la modernización del Estado, algunas Universidades Públicas en Chile han desarrollado programas de formación profesional de trabajadores del sector público, dirigidos a desarrollar competencias y habilidades profesionales a funcionarios de la administración pública en casi todas las regiones de Chile. Si bien ambos esfuerzos parecen vinculados, por los objetivos y tópicos que involucran, se carece de investigaciones empíricas que evalúen el impacto de los repertorios discursivos del Estado hacia sus funcionarios para llevar adelante la modernización, así como el impacto de los procesos de formación profesional dirigidos a trabajadores del sector público. Este trabajo se sitúa en este vértice analíti co. Analiza y evalúa el rol formativo de la Universidad, a partir de la experiencia de la Universidad de Los Lagos que entre 1994 y 2000 ha formado a más de 600 profesionales, que iniciaron sus estudios siendo trabajadores públicos, relacionándolo con el proceso de modernización del Estado en Chile. Para ello, son consideradas como unidades de información y análisis los trabajos finales de titulación de los estudiantes adscritos a programas de formación profesional para trabajadores de la administración pública, ya que estos trabajos reflejan la respuesta de los funcionarios públicos hacia el proceso de modernización.

Palabras clave: Formación profesional, modernización del Estado, políticas públicas.

Recibido: 01-11-14 . Aceptado: 02-04-10

* Administrador Público, Magíster en Estudios Internacionales. Director del Departamento de Gobierno y Empresa del Campus Puerto Montt de la Universidad de Los Lagos, Chile. E-mail: ndiaz@ulagos.cl

** Licenciado en Educación, Magíster (C Ciencias Políticas. Director Red de Servicios Universitarios Regionales-Santiago de la Universidad de Los Lagos, Chile

*** Administrador Público, Coordinadora Programa de Extensión Académica Universidad de Los Lagos-Santiago, Chile 


\section{New Strategies in Profesional Training to Promote State Modernization in Chile}

\section{Abstract}

Since the beginning of the 1990s, there has been a growing interest in modernization on the part of the Chilean government. This change is evident in diverse policies, programs and institutions, and could together be articulated as the modernization of public policy which has been promoted by public officials, and which gives emphasis to the role that human resources plays in the success of such a process. In a convergent effort towards state modernization, certain public universities in Chile have developed professional training programs for public sector employees, directed towards developing professional competence and ability in public administration officials in almost all regions of the country. Even though both processes would seem to be related due to the objectives and topics covered, there has been no empirical research to evaluate the impact of the rhetorical discourse of the state on public officials in order to modernize the state, nor has there been an evaluation of the impact of these professional programs directed towards state officials. This paper is of an analytical nature and analyzes the formative role of the university, based on the experience of the Los Lagos University between 1994 and 2000, in the formation of over 600 professionals who initiated their studies as state functionaries in conjunction with the process of state modernization in Chile. As subjects of the study and units of information, the final thesis of those who finished the program of professional formation for public administrators and workers were considered, since these thesis reflect the recommendations of these public officials in relation to the process of modernization.

Key words: Professional formation, state modernization, public policy.

\section{Introducción}

En la última década se aprecian en Chile procesos convergentes en torno a la formación de recursos humanos para la modernización del Estado. De una parte, se observa el surgimiento y desarrollo de programas de formación profesional y técnica, dirigidos hacia funcionarios públicos; resultado de importantes transformaciones sociales y educativas, de una mayor valoración de la educación como vehículo de promoción social, la demanda de organizaciones e instituciones hacia las universidades para intervenir en la definición de programas profesionales y la incorporación de nuevos segmentos de la población a las universidades.

De otra parte, los esfuerzos de política pública para la modernización del Estado, confieren a los recursos humanos un papel relevante. Esfuerzo que subraya no sólo la inclusión del área de recursos humanos como línea de intervención para el mejoramiento de la gestión pública; también porque han ganado protagonismo en otras transformaciones planteadas como objetivo de política pública para los próximos años. Como en: mejoramiento de la calidad y la gestión de servicios, participación ciudadana, auditoria, transparencia y probidad en la gestión y, 
la incorporación de tecnologías de información y comunicación. En todas ellas también mayores calificaciones de recursos humanos.

El presente trabajo intenta responder las siguientes preguntas directrices:¿cuáles son los elementos analíticos que explican el desarrollo de programas de formación profesional para funcionarios de la administración pública en Chile?, y ¿qué relaciones existen entre las competencias profesionales adquiridas en los programas de formación profesional para funcionarios de la administración pública, los intereses profesionales de los funcionarios-estudiantes y los repertorios discursivos del Estado en su proceso de modernización?. La primera pregunta, será abordada analizando las principales tendencias y cambios experimentados en los sistemas de formación profesional y, una experiencia de programa de formación profesional y técnicas desarrollada por la Universidad de Los Lagos para funcionarios públicos. La hipótesis de trabajo sugerida es que los programas de formación profesional para funcionarios pú blicos, se explican por la necesidad de elevar los niveles de profesionalización en el Estado para hacer frente al proceso modernizador y los nuevos paradigmas de la formación profesional.

La segunda interrogante, será enfrentada a través del análisis y evaluación de proyectos finales de titulación realizados por funcionarios para acceder a un título profesional. Estos proyectos pueden ser considerados como una síntesis entre las competencias adquiridas, los intereses profesionales y los repertorios discursivos del Estado en torno a su proceso de modernización. La hipótesis, en esta dimensión, es que las áreas y líneas de intervención de los proyectos finales de titulación están relacionadas con los esfuerzos de política pública para la modernización del Estado en Chile.

\section{Capital Humano y Transformaciones en la Formación Profesional}

Existe consenso desde hace décadas sobre el papel relevante de la educación como factor de desarrollo de los países y los individuos ${ }^{1}$. Así el debate político actual establece como supuesto que la inversión en capital humano y un elevado nivel de competencias entre la población activa ejercen un impacto benéfico mas 0 menos directo sobre el rendimiento económico y la competitividad de los países (Bachtemann y Sodoff, 1998). Ello ha llevado a los Estados nacionales a establecer políticas de promoción educacional y centrar sus esfuerzos en la expansión de las coberturas educativas, el mejoramiento de los sistemas educacionales y

1 Desde los trabajos de Gary Becker sobre capital humano a mediados de los 60, una enorme literatura ha encontrado evidencias sobre la asociación entre niveles educativos de la población y crecimiento económico. El tema ha ido copando la agenda de los organismos multilaterales de financiamiento y cooperación como el Banco Interamericano de Desarrollo, Banco Mundial, Fondo Monetario Internacional y UNESCO como una estrategia de crecimiento económico para los países en desarrollo. Ver: Banco Mundial (2000); UNESCO (1997); Banco Mundial (1993) y Jacques (1996). 
el desarrollo de sistemas de investigación y desarrollo tecnológico.

Un conjunto de tendencias que se observan en la formación profesional, parecen converger en la búsqueda de nuevas alternativas que eleven y mejoren el capital humano de los países en desarroIlo. Entre estas tendencias se encuentran:

a) Aumento de su cobertura. Según la UNESCO los estudiantes de educación superior en América Latina aumentaron entre 1970 y 1991 de 2 a 8 millones, y a nivel mundial pasará de 65 millones en 1991 a 100 millones en el 2005. A pesar de este notable crecimiento, todavía existen importantes diferencias en las oportunidades de acceso a la educación superior. Mientras en la Unión Europea y Estados Unidos, la tasa de escolarización en educación superior es de 60 por cien, en América Latina es de 20 por cien (De Moura, 2000).

b) Incorporación de nuevos segmentos de la población. Los tradicionales estudiantes jóvenes egresados de la educación secundaria han dejado de ser el grupo etáreo exclusivo de las instituciones de formación profesional. Crecientemente se han incorporado nuevos grupos sociales, como los trabajadores que combinan estudios y trabajo. En los Estados Unidos más de la mitad de la población estudiantil está compuesta en el nivel terciario por alumnos maduros y de tiempo parcial. Finlandia, tiene 150 mil estudiantes regulares de educación superior y 200 mil adultos inscritos en programas de enseñanza continua (Brunner, 2000). Esta tendencia también se observa en América Lati- na, facilitada por el desarrollo de nuevos programas de educación continua y a distancia.

c) Nuevos actores y orientación hacia la demanda. Nuevas organizaciones públicas y privadas solicitan a las universidades programas de formación profesional orientados hacia competencias profesionales específicas. Ello configura una red de diálogo que interviene en la definición y planificación de los programas de formación profesional, desplazando la gravitación de las universidades como agente exclusivo en el rol formativo (Leibowics, 2000). Esta tendencia está relacionada con un proceso más complejo de redistribución de capacidades para generar y administrar conocimiento, que han dado lugar a una red compleja donde interactúan: universidades, institutos de formación técnica, fundaciones, instituciones públicas, empresas y organismos no gubernamentales.

d) Superación de los ciclos formativos clásicos. Los ciclos formativos úni$\cos$, donde se adquieren competencias para toda la vida laboral están siendo superados por la velocidad de los cambios observados en todas las áreas profesionales. La nueva forma de entender la educación es para toda la vida; los profesionales de manera creciente se encuentran adscritos a programas regulares de formación y especialización.

Los países en desarrollo están convencidos que elevando los niveles de formación profesional de la población, aumentarían sus posibilidades de competir en una economía abierta, pero carecen 
de los recursos y sistemas nacionales de formación profesional, que puedan avanzar más rápidamente en el aumento de las coberturas educacionales y el mejoramiento de los sistemas de formación profesional. Sin embargo, de manera creciente, y en algunos casos sin el auspicio del Estado, se están desarrollando experiencias nuevas que podrían contribuir al aumento del capital humano.

La formación profesional de trabajadores aparece como una alternativa viable de desarrollo de capital humano. Atendiendo a: el mantenimiento de la distancia relativa entre países desarrollados y en desarrollo, respecto de las tasas de escolarización superior; la valoración de la educación como vehículo de promoción social; la flexibilización creciente de los programas de formación profesional; el cofinanciamiento público y privado de la formación y el desarrollo de tecnologías de la información, la formación profesional de trabajadores sería una contribución decisiva para las sociedades en desarrollo.

La formación profesional enfocada desde una perspectiva laboral, tiene su aplicación en distintas situaciones del individuo que hace uso de ella:

a) Formación de jóvenes, en las técnicas o habilidades que le permitan su incorporación al mundo laboral. Se pueden incluir la formación profesional y capacitación, desde materias muy específicas, hasta más genéricas o globales.

b) Formación dirigida a grupos con experiencia en el mundo laboral, que atraviesan una situación de desocupación o con problemas de inserción que buscan nuevas alternativas pro- fesionales, de perfeccionamiento o de reconversión laboral, y

c) Formación de trabajadores en situación laboral, con la finalidad de mejorar su capacidad profesional, bien en el puesto de trabajo o bien con la finalidad de promoción.

En general, el proceso de formación profesional está en función de al menos cuatro criterios: su vinculación con el sistema de relaciones laborales, como parte de los procesos de transferencia tecnológica; como hecho educativo, y se orienta hacia la estructuración de competencias (CINTERFOR, 1996). La formación profesional de trabajadores tiende a extenderse como un complejo sistema que articula la incorporación de trabajadores a sistemas tradicionales de formación profesional, y más recientemente al surgimiento y desarrollo de la formación continua, como un sistema cada vez más estructurado y masificado en los países desarrollados. Por formación continua se entiende el proceso mediante el cual los individuos encuentran no sólo los elementos para ingresar al mercado del trabajo, sino las capacidades para transitar todos los pasajes que sean necesarios para diferentes empleos y exigencias laborales.

Este nuevo sistema de formación ha ido adquiriendo mayor atención de parte de los gobiernos, las empresas y las organizaciones de trabajadores. En España, en 1992 y 1996 fueron firmados y renovados Los Acuerdos Nacionales de Formación Continua, acuerdos tripartitos que establecen una estructura jerárquica y financiera del sistema. En Francia, la legislación sobre formación profesional ha sido objeto de numerosas reformas en los 
últimos años. Actualmente dispone que las empresas deben responder con el $1,5 \%$ de las remuneraciones brutas destinadas a formación. Esta cifra, es distribuida en aportes de capacitación para jóvenes en el sector público (necesaria en el proceso de ingreso al mercado de trabajo), permisos individuales de formación para los trabajadores y programas de formación y capacitación contratados libremente por la empresa. Desde 1972 la proporción de trabajadores franceses que reciben servicios de capacitación y formación se ha elevado del $8 \%$ al $30 \%$. En Francia las leyes han servido de estímulo para el aporte de las empresas, doblando las exigencias de la inversión que les impone la ley (Martínez, 1999). Instrumentos legales y fondos públicos destinados a promover la formación profesional y la capacitación, se encuentran ampliamente desarrollados también en Italia, Alemania, Corea y Japón, por citar sólo algunos países (Rivera y Neira, 2001).

En la búsqueda de una definición comprensiva aplicable a Chile -probablemente también a otros países en desarrollo- puede considerarse al proceso de formación profesional de trabajadores como el sistema integrado de acciones conducentes a la transformación de potencialidades de los trabajadores que permite una mayor y mejor efectividad en el desempeño de sus actividades laborales. Una síntesis sobre los impactos potenciales de un sistema formativo de este tipo supondría para el trabajador (CON. FEMETAL, 1999):

- Adquirir conocimientos, destrezas, habilidades.
- Integrarse a un equipo organizacional.

- Conocer nuevas tecnologías y formas de trabajar para "mantenerse" y "conservar" su puesto de trabajo.

- Desarrollo personal y profesional (promoción, status, salario).

- Fortalecer el reconocimiento de sus compañeros de trabajo (ser escuchado, ser valorado).

- Contribuir de mejor forma al "proceso productivo" de la organización, a través de la generación de nuevas ideas.

- Aumentar su efectividad y eficiencia en su área.

- Aplicar inmediatamente los nuevos recursos tecnológicos aprendidos a las necesidades de la organización.

Para la organización:

- Proporcionar un aliado estratégico para el cumplimiento de su misión.

- Incorporar valor agregado al recurso más determinante de su quehacer.

- Mejorar el clima laboral.

- Aumentar los niveles de competitividad, productividad, rentabilidad y calidad.

- Disponer en los cargos de la organización de personal calificado e integrado en la cultura organizacional de la empresa.

- Desarrollar las potencialidades de los equipos de trabajo.

- Permitir mayor ductibilidad para los cambios del entorno.

Otro concepto que articula la necesidad de integrar la formación con las transformaciones sociales, es el de formación a lo largo de toda la vida (CINTERFOR, 1998). La incorporación de 
esta matriz conceptual y teórica tendría como consecuencias: un desplazamiento hacia la formación general que desarrollen habilidades y capacidades en un mundo laboral en transformación, la responsabilidad compartida en la formación donde interactúan diferentes instituciones e individuos, y una oferta flexible de programas de formación.

Este debate se ha centrado en la actualidad en dos temas centrales: el primero, referido a la necesidad de reformular el concepto de formación vigente de acuerdo con los requerimientos de los sistemas laborales, productivos y educativos; el segundo, relacionado con el diseño de una nueva institucionalidad para la formación que recoja el impacto que significa la aparición de nuevos paradigmas $y$ actores en el marco del sistema formativo profesional.

\section{La Modernización del Estado en Chile}

El proceso de modernización del Estado en Chile ha estado orientado a introducir una cultura organizacional equivalente a las necesidades de desarrollo económico y la democratización, con niveles cada vez más elevados de bienestar y en condiciones de mayor igualdad de oportunidades. Estas orientaciones de política pública en materia de modernización se ven reflejadas en los programas y las acciones de los gobiernos, que desde 1990 han estado sustentadas en lo siguiente:

a) Se aparta del modelo de Estado interventor y de las concepciones conservadoras que pretenden restringir su ámbito de acción al mínimo posible.
Al Estado que se apunta es uno que sea capaz de buscar fórmulas innovadoras que permitan la cooperación entre el sector público y el sector privado, de modo de movilizar al máximo posible los recursos humanos $y$ materiales de la sociedad.

b) Se considera al Estado como un instrumento al servicio de las personas; el Estado puede ser sustituido en sus funciones sin desmedro de la calidad de los servicios.

c) Se le asigna al Estado una función normativa y de regulación; para el Estado, una función importante es aquella que puede ser descrita como la función de regulación de los mercados, tanto a partir de objetivos de logro de los mejores niveles de eficiencia en la operación de los mismo mercados, como de otros originados en la evolución de la sociedad y en las sensibilidades colectivas que en ella cristalizan, tales como la defensa del medio ambiente, los recursos naturales y la protección de la vida urbana.

d) Se le confiere al Estado un rol preponderante en la tarea de impulsar la equidad y garantizar la cohesión social; el Estado debe impulsar políticas de subsidio, salud, seguridad social, educación, vivienda, capacitación y reconversión tanto laboral como geográficas.

e) Se considera que un Estado más eficaz y eficiente es condición necesaria para velar por la equidad, estabilidad, coherencia, competitividad y sustentabilidad del proceso de desarrollo; el Estado debe hacer un esfuerzo permanente por mejorar y potenciar las ventajas adquiridas y en un clima de unidad, estabilidad, innovación y 
creatividad y generar otras que permitan a futuro profundizar la estrategia de desarrollo que se ha elegido.

La tradición administrativa chilena ha creado una cultura institucional que se vincula a un Estado clásico, con funciones esencialmente políticas y normativas, con una estructura autoritaria y jerárquica, un sistema vertical y unilateral de decisiones, y un estilo de administración que pone el acento en los procedimientos y los métodos, antes que en los resultados. Los órganos de la administración del Estado, están sujetos al principio de la legalidad, es decir, no tiene atribuciones que no les permita la Ley, están sujetos a la fiscalización política de la Cámara de Diputados y al control de juridicidad de sus actos que realiza la Contraloría.

En el contexto anterior, la administración pública se presenta como un sistema rígido y reglamentado, con un alto nivel de centralización, lo que conlleva un sector público muy poco dinámico, con niveles de adaptación muy lentos, donde los cambios anteceden rápidamente a las condiciones creadas para asumirlos, creándose una constante necesidad de cambio que se encuentra sujeta a los vaivenes de los desafíos que se le presentan.

En la actualidad, las políticas públicas de modernización del Estado están orientadas y canalizadas institucionalmente en el Proyecto de Reforma y Modernización del Estado ${ }^{2}$. Entre sus princi- pales líneas de acción se encuentran el impulso de siete reformas generales del Estado chileno: Reforma y modernización del Estado; Reforma tecnológica, Reforma de la ciudadanía, Reforma fiscal, Reformas políticas, Reformas del trabajo, Reformas de la salud y la continuación de la Reforma judicial y educacional. El proyecto también se propone áreas específicas de intervención para el mejoramiento de la gestión pública, tales como recursos humanos, calidad del servicio, gestión de servicio, participación ciudadana, auditoria, transparencia y probidad de la gestión, y tecnologías de información y comunicación.

En el área recursos humanos, se plantea el nuevo trato para funcionarios públicos y la dirección pública. Los principales temas a tratar son el estatuto de dirección pública, su implementación en 33 servicios, el sistema de formación de directivos públicos y la preparación del proyecto de ley sobre la materia. De acuerdo a ésta línea de trabajo se contempla un programa de capacitación, radicado en las Universidades autónomas que postulen a un sistema de acreditación que permita evaluar la calidad y pertinencia de los programas formativos a través de una agencia independiente.

Las características del sector público en Chile, dan cuenta de las condiciones en que se encuentra el sector público frente a los cambios sociales y económiministerial de Modernización de la Gestión Pública bajo el Gobierno de Eduardo Frei Ruiz Tagle (1994-2000). 
cos que toca enfrentar. El número de funcionarios públicos, en un sentido amplio, tomando en cuenta al personal civil, está compuesto por más de 130 mil personas que trabajan a nivel de gobierno central, los que comprenden el 2,5\% del empleo total del país. Agregando los municipios se llega al $3 \%$. Ello representa un $5,5 \%$ de la fuerza de trabajo empleada en el país. La profesionalización del sector público llega a un $25 \%$. Los dos tercios del personal son de planta, ello significa que se rigen por todas las normas y beneficios que incluye en el sistema laboral para el sector público. Un tercio pertenece a un régimen mucho más flexible, debido a que trabajan a contrato de un año y, por lo tanto, a condiciones de movilidad y estabilidad radicalmente distintas al resto de los trabajadores del sector público. Las remuneraciones están basadas en una escala única. Los ascensos, calificaciones y retiros se encuentran determinadas por el Estatuto Administrativo (Vial, 1996).

Estos antecedentes sugieren que el éxito en la implementación de políticas de modernización del Estado, en una medida importante depende de la capacidad del propio Estado para mejorar las competencias técnicas y profesionales de sus funcionarios. Sin embargo, se carece de instrumentos y estímulos directos para alentar la formación de los funcionarios. La incorporación de funcionarios de la adminis- tración pública en programas de formación profesional y técnica de las universidades en Chile, obedece más el esfuerzo individual por mejorar el status social y sus condiciones de vida, que a iniciativas planificadas desde los servicios públi$\cos ^{3}$.

\section{Formación Profesional para la Administración Pública en Chile}

Dos corrientes distintas son las que han predominado históricamente en la formación en el campo de la administración pública. Por una parte, la tradición Europea ha propendido a formar a los servidores públicos en Centros de Desarrollo Funcionarial del Estado, destacando La Escuela Nacional de Administración en Francia, La Escuela de Ciencia de la Administración de Spira en Alemania, el Instituto Nacional de Administración Pública en España y el Colegio del Servicio Civil en el Reino Unido. En cambio, tanto en la tradición iberoamericana como en Estados Unidos, la formación de gerentes públicos ha estado ligada a la enseñanza universitaria.

La mayoría de las tradiciones regionales relativas a la formación en administración pública tienen una raigambre similar: la enseñanza ofrecida por las instituciones académicas europeas a partir de 1727, cuando los cursos de ciencia de

Uno de los inconvenientes de los Servicios Públicos en Chile para apoyar a los funcionarios a través de destinaciones de estudios y financiamiento, es la omisión de la formación profesional, como vehículo de promoción en el Estatuto Administrativo, que es el cuerpo jurídico que regula las relaciones del Estado con los funcionarios públicos. En el Estatuto Administrativo no está prevista la Capacitación como instrumento de mejoramiento de los recursos humanos 
la Administración- ciencias cameralesfueron introducidos en las Universidades de Halle y Frankfurt del Oder en Alemania, a través de la profesión en Economía, Policía y Cameralística. Esta experiencia docente fue fructífera y longeva, todavía se enseñaban ciencias camerales a mediados del siglo XIX en las aulas de las Universidades de Viena y Tubinga (Guerrero, 1992). La experiencia universitaria cameralista fue el origen de los modernos cursos de formación funcionarial ofrecidos por el Estado, cuyos inicios fueron muy difíciles: La Escuela Especial de Madrid y la Escuela de Administración de París, establecidas respectivamente en 1842 y 1848 , cerraron sus puertas poco tiempo después de la inauguración de sus cursos y se debió esperar hasta mediados del siglo XX para que cristalizara efectiva y permanentemente en los centros de desarrollo funcionarial de Estado.

En el caso de Chile, a partir de la instauración del régimen desde 1973, una progresiva crisis se acentuó en la visión de como debía institucionalizarse la relación entre el Estado y la Sociedad. La intensiva penetración del Estado en casi todas las áreas de la Economía que se verificaban hasta 1973, no fue vista por régimen militar como una situación coyuntural, producto de la aplicación de un proyecto político socializante, sino como la culminación de un largo proceso de expansión estatal (Mancilla, 1984). La visión crítica del régimen militar sobre el rol del Estado, al que se atribuía ser causante de las bajas tasas de crecimiento del país entre las décadas de 40 y 70 , no obedecía a un enfoque económico y parcial, sino a una perspectiva ideológica y globalizante (Díaz, 1996).
Estas apreciaciones influyeron en forma determinante en los procesos formativos de profesionales ligados al quehacer del Estado que estaban desarrollándose en universidades chilenas y, claramente, en los procesos de capacitación del recurso humano que trabajaba en el Estado. En general, el recurso humano fue el factor más abandonado del proceso reformador del Estado llevado a cabo por el gobierno militar; la capacitación y el adiestramiento fueron reducidos drásticamente. Inclusive en La Comisión Nacional de Reforma Administrativa (CONARA), se llegó a estudiar un detallado reglamento de calificación de Personal, que finalmente se abandonó, pues habría resultado una inconsecuencia enfatizar o anteponer la calificación que formaliza sanciones y estímulos sobre la capacitación que creaba las condiciones para que tales sean efectivos (Mancilla, 1984).

Una de las pocas modificaciones sustantivas introducidas en el área específica de los recursos humanos fue la creación de la Escala Única de Remuneraciones, la cual uniformó inicialmente todas las remuneraciones de la administración pública central y municipalidades, para llevar a la práctica el principio de equidad: es decir a igual función debería corresponder igual salario. La Escala Única de Remuneraciones introdujo una fuerte compensación a los estudios superiores por la vía de la llamada "Asignación de Título" o "Asignación Profesional”, que es un porcentaje variable que se agrega al sueldo base. No obstante, en el largo plazo no hubo ningún impacto efectivo en la capacitación sistemática de los estratos medios y altos de la administración pública (Mancilla, 1984). 
En Chile, no existe tradición de institutos gubernamentales para la formación de los altos funcionarios públicos, ya que la opción ha sido entregar esta misión al sistema universitario, por corresponder a la misión de la Universidad la formación de las elites de la sociedad ${ }^{4}$. Todavía más, existen antecedentes fundados de que esta función formativa de los profesionales de la administración pública, seguirá radicada en los próximos años en el sistema universitario.

El sistema universitario en Chile incluye 16 universidades estatales y 49 universidades privadas; existen 8 escuelas de formación de administradores públicos, que reciben indistintamente el nombre de Escuelas de Ciencias Políticas y Administrativas o Escuela de Administración Pública a excepción de la Universidad de Chile que a partir de 1995 se denomina Escuela de Gobierno y Gestión Pública. Las más antiguas son las Escuelas de la Universidad de Chile y de la Universidad de los Lagos. Se encuentran distribuidas en una extensa zona geográfica, desde Antofagasta a Puerto Montt. Todas poseen un curriculum relativamente similar. Son 5 las Escuelas que tienen su sede en la ciudad de Santiago.

Todas las Escuelas realizan una función docente, que consiste en la for- mación de profesionales a nivel de licenciatura, con cinco años de duración después de la enseñanza media, incluyéndose en todas ellas asignaturas de administración y gestión, jurídicas, económicas, ciencia política y sociología, y demás ramos complementarios.

\section{La Formación Profesional de Trabajadores de la Administración Pública}

Desde mediados de los 90 la Universidad de Los Lagos implementa Programas de Formación de Trabajadores ${ }^{5}$. Estos programas se diferencian de los regulares de formación profesional por su forma de ingreso, áreas de formación profesional, extensión de ciclos formativos, destinatarios y financiamiento (cuadro1). En este marco un programa especial ha estado dirigido a la formación profesional de trabajadores de la administración pública: las carreras de Ingeniería de Ejecución en Administración Pública y Técnico Universitario en Administración Pública (Universidad de los Lagos; 1994; 1995). Inicialmente, esta idea responde a un planteamiento realizado por la Asociación Nacional de Empleados Fiscales (ANEF), en orden a revertir el histórico abandono sufrido por los funcionarios pú-

4 En julio del presente año se inició un proceso de acreditación de los programas de formación y especialización en Gestión Pública, por parte del Proyecto de Modernización del Estado y el Consejo Superior de Educación. Si bien el proceso está orientado inicialmente a la formación de postgrado y la alta especialización, parece una señal clara del Estado para mantener la formación en el sistema universitario, incorporando elementos evaluativos y regulatorios a través de instituciones que cautelen la fe pública.

5 La Universidad de Los Lagos es una de las 16 universidades públicas en Chile y cuenta en la actualidad con 15.000 estudiantes en cuarenta sedes en Chile. De este número, un 65\% corresponde a estudiantes inscritos en programas de formación profesional de trabajadores 


\section{Cuadro 1 \\ Universidad de los Lagos \\ Comparación entre el Programa de Formación Profesional Regular y El Programa de Formación Profesional de Trabajadores de la Universidad}

\begin{tabular}{|c|c|c|}
\hline Dimensión & $\begin{array}{l}\text { Programas de Formación Profe- } \\
\text { sional Regulares }\end{array}$ & $\begin{array}{l}\text { Programas de Formación Profe- } \\
\text { sional de Trabajadores }\end{array}$ \\
\hline Forma de ingreso & $\begin{array}{l}\text { Los estudiantes ingresan dando } \\
\text { un examen nacional (Prueba de } \\
\text { Aptitud Académica) }\end{array}$ & $\begin{array}{l}\text { Los estudiantes ingresan acredi- } \\
\text { tando su condición de trabajado- } \\
\text { res }\end{array}$ \\
\hline $\begin{array}{l}\text { Áreas de formación } \\
\text { profesional }\end{array}$ & $\begin{array}{l}\text { Administración, Ciencias Socia- } \\
\text { les, Educación, Ingeniería, Acui- } \\
\text { cultura, Arquitectura, Alimentos, } \\
\text { Contabilidad }\end{array}$ & $\begin{array}{l}\text { Administración, Educación Cien- } \\
\text { cias Sociales, Informática }\end{array}$ \\
\hline $\begin{array}{l}\text { Extensión de ciclos } \\
\text { formativos }\end{array}$ & $\begin{array}{l}\text { Ciclos formativos largos centra- } \\
\text { dos en el aprendizaje inicial }\end{array}$ & $\begin{array}{l}\text { Ciclos formativos intermedios } \\
\text { orientados al aprendizaje y la } \\
\text { práctica laboral }\end{array}$ \\
\hline Destinatarios & Jóvenes entre 18 y 25 años & Mayores de 25 años \\
\hline Financiamiento & Público y privado & Privado \\
\hline
\end{tabular}

Fuente: Elaboración Propia

blicos en Chile. La iniciativa, contó también con el auspicio del Gobierno a través de la Subsecretaría de Desarrollo Regional y Administrativo del Ministerio del Interior.

En consideración de los costos financieros que implicaba la matrícula y el arancel para funcionarios públicos, con bajos niveles de remuneración, se materializó un convenio entre la ANEF y el Banco del Estado, mediante el cual este Banco financia el $50 \%$ del valor del arancel anual, con un crédito blando, suscrito por el funcionario público y pagado automáticamente a la Universidad. Este hecho es la primera experiencia de financiamiento bancario en la Educación Supe- rior en Chile. Paralelamente, la Universidad de Los Lagos firmó más de 40 convenios de colaboración para favorecer el ingreso de los funcionarios a los programas de formación profesional ${ }^{6}$, lo que representó una ayuda económica adicional para los trabajadores.

En su génesis, el Programa se inició simultáneamente en la Novena región de la Araucanía (Temuco), en la X región de Los Lagos (Osorno y Puerto Montt), y en la XI región Aysén del General Carlos Ibáñez del Campo (Coyhaique). Posteriormente fue extendido desde la cuarta región de Coquimbo hasta la Duodécima región de Magallanes y la Antártica Chilena, con presencia en todas las capitales miales y de funcionarios del estado, que consideran descuentos en los valores de las colegiaturas. 
regionales circunscritas entre las regiones mencionadas, con una matrícula total de 1.200 funcionarios públicos.

El Programa de formación profesional se propone los siguientes objetivos:

a) Formar un profesional capacitado para formular, implementar y ejecutar políticas, planes, programas y proyectos como parte de los programas de desarrollo en las instituciones del sector público.

b) Desempeñar posiciones ejecutivas y técnicas en los diferentes niveles y sectores de las organizaciones y sistemas gubernamentales y administrativos, tanto en el ámbito del Gobierno y la Administración Central como Regional y Local.

En términos específicos, el Programa pretende que los egresados:

a) Comprendan las ideas del desarrollo socioeconómico y cómo se relaciona con las concepciones políticas y administrativas de los asuntos del Estado.

b) Detecten los requerimientos de cambio y progreso provenientes de la propia administración del Estado y del medio en que está inserto.

c) Reconozcan problemas particulares de la administración, particularmente de la administración del Estado y sean capaces de incorporar los avances científicos y tecnológicos de la administración a la realidad concreta donde se desempeñan. d) Sean capaces de impulsar la excelencia en el ejercicio de la función pública administrativa.

e) Ejerzan la función administrativa teniendo en consideración los principios éticos y morales de la sociedad y particularmente las inherentes a la función pública.

El programa se articula sobre la base de una formación científico-técnica en las áreas socio-política, económica, jurídica y administrativa. La formación multidisciplinaria le permite una visión integrada de los problemas de la sociedad. Los contenidos del Programa de Estudio, posibilita el desarrollo de capacidades para la creación e implementación de soluciones nuevas, de acuerdo a las indicaciones del interés nacional.

Las nuevas tendencias en los programas de estudio buscan acercar la formación teórica con la experiencia ${ }^{7}$, mientras el desempeño laboral de los estudiantes del programa de formación profesional para trabajadores públicos supone resolver la condicionante de este obstáculo, quedando pendiente como foco de interés analítico la canalización pedagógica de esta experiencia en el proceso formativo. Ello cobra más sentido, al no considerar a la administración pública como una disciplina, sino como una práctica social y una profesión. Desde otra perspectiva, la articulación entre teoría y práctica, entre conocimiento académico y

$7 \quad$ Una de las preocupaciones en Estados Unidos es la forma de robustecer la experiencia laboral en los programas de formación para la Administración Pública. Scholl (1995), sugiere para ello tres estrategias: acceso a oportunidades dentro de la sala de clases, ejercitar las habilidades y conocimientos aprendidas en la clase, y hacer experiencia fuera del aula de clases. 
conocimiento profesional, sin subordinaciones mutuas, serían necesarias para la formación de teorías que amplíen el conocimiento sobre la gestión pública. Mientras el conocimiento académico, facilitará en los programas formativos la comprensión de los niveles marcos del universo ecológico de la gestión pública, los conocimientos derivados de la práctica profesional ayudan a comprender los niveles micro del mismo universo, como son el quehacer administrativo, las organizaciones públicas, las políticas públicas (Ospina, 1998).

El Programa tiene una duración de ocho semestres académicos, en su fase lectiva, y es conducente al título profesional de Ingeniero de Ejecución en Administración con mención en Administración Pública. En 6 semestres, es conducente título de Técnico Universitario en Administración Pública. Cada semestre académico puede ser considerado como módulo de capacitación. Las actividades curriculares previstas en el programa de estudio se desarrollan en horario vespertino, tres veces a la semana y sábado en la mañana, lo que permite a los funcionarios compatibilizar su actividad laboral, estudiantil y familiar.

Los estudiantes expresan una percepción favorable al Programa de Formación Profesional. Investigaciones realizadas revelan que el $75,5 \%$ de los alumnos del Programa de Ingeniería (e) en Administración Pública consideran que los es- tudios realizados han sido satisfactorios, en tanto el $76,5 \%$ considera que el programa de estudios le ha permitido mejorar su desempeño laboral ${ }^{8}$ (Universidad de Los Lagos, 2000). Otro indicador de satisfacción de los estudiantes por el Programa de Estudios es su disposición a recomendar a otros compañeros de trabajo incorporarse al Programa. El 86,4\% de los encuestados manifestó que recomendaría a otras personas ingresar a la carrera que cursan.

Las mismas investigaciones informan sobre el alto sentido de pertenencia de los estudiantes con las instituciones públicas de origen. El $86,4 \%$ de los encuestados desea permanecer en su institución o servicio público una vez finalizada su carrera. Ello sugiere una alta valoración de los estudiantes por su trabajo y las condiciones para la permanencia en el sector público de los profesionales una vez titulados.

\section{Análisis de los Proyectos Finales de Titulación}

Los resultados corresponden a la primera etapa de una investigación destinada a evaluar el impacto del programa de formación profesional de trabajadores del sector público que imparte la Universidad de Los Lagos en Chile. En esta primera etapa se entregan resultados del análisis realizado a los Proyectos Finales de Titulación (PFT) de los estudiantes de

8 El estudio fue realizado en el año 2000, como parte de un sistema de seguimiento de los Programas de Formación Profesional de Trabajadores. Los resultados representan una muestra del $60 \%$ de los estudiantes de Ingeniería (e) en Administración Pública en 6 sedes donde se imparte el Programa 
los programas de formación profesional para trabajadores del sector público.

Los PFT corresponden al requisito académico final del currículo formativo. Su propósito es articular los dominios conceptuales y competencias profesionales recibidas en la formación, con la experiencia laboral en la administración pública. Los estudiantes, en este marco, realizan una práctica profesional y producen un informe final sometido a evaluación ${ }^{9}$.

El análisis de los PFT puede entenderse como una síntesis que articula: aprendizajes del programa formativo, motivaciones de interés profesional y los repertorios discursivos del proceso de modernización del Estado. De modo específico, la investigación que se presenta tiene los siguientes objetivos:

a) Identificar las áreas de intervención de los PFT

b) Comparar las áreas del Programa de Estudio de la carrera de Ingeniería (e) en Administración Pública presentes en los PFT.

c) Analizar la relación existente en las áreas de los PFT con los esfuerzos de políticas públicas e instrumentos del proceso de modernización del Estado

Fueron consideradas 185 PFT de estudiantes titulados en las sedes de La Serena, Valparaíso, Santiago y Puerto Montt ${ }^{10}$. Los trabajos fueron revisados y clasificados en diferentes áreas de intervención. Del universo de los trabajos, fue obtenida una muestra aleatoria estratificada ( $20 \% ; n=39)$, sobre la cual se realizó un trabajo de inducción analítica (Goetz, 1998). Una vez levantadas las categorías de trabajo, los PFT fueron resumidos a pautas de trabajo que contenían las siguientes dimensiones: datos de identificación (nombre, institución pública), área de intervención, tema, áreas de formación del programa de estudios, áreas de gestión pública, objetivos y resumen.

\section{1 Áreas de Trabajo en los Proyectos Finales de Titulación}

Los PFT representan áreas diversas de intervención. Mayoritariamente están referidos a Estudios, Procesos de Operacionalización y Racionalización y Recursos Humanos. Estas tres áreas concentran el $51,8 \%$ de los proyectos analizados (Tabla 1). Los PFT referidos a estudios incluyen áreas tales como Jurídicos, Políticas Públicas, Institucionales y Teóricos. Estos últimos relacionados con algún tema de interés para los estudiantes, relacionados con algunas de las áreas disciplinarias presentes en el Programa de Estudio. Los PFT en Operacionalización y Racionalización están dirigidos a mejoras en la organización a través

9 La práctica profesional y el informe final poseen una ponderación del 25\% de la calificación final del programa y otro $25 \%$ la defensa del Informe ante una comisión académica.

10 Las cuatro sedes consideradas fueron escogidas de entre doce sedes por su tamaño: dos de un alto número de titulados (Santiago y Puerto Montt) y dos de un reducido número de titulados (La Serena y Valparaíso), y por su ubicación geográfica: dos de la zona central (Santiago y Valparaíso) y dos regiones alejadas de la capital (La Serena y Puerto Montt). Los 185 PFT se distribuyen de la siguiente manera entre las sedes estudiadas: La serena (22), Santiago (84), Valparaíso (37) y Puerto Montt (42). 


\section{Tabla 1}

Universidad de los Lagos

\section{Programa de Formación Profesional para el Sector Público Áreas de Trabajos Finales de Titulación por Sedes (En \%)}

\begin{tabular}{lccccc}
\hline Área & Santiago & Puerto Montt & La Serena & Valparaíso & Totales \\
Estudios & 29,7 & 12,0 & 9,0 & 18,9 & 21,1 \\
Operacionalización & & & & & \\
y Racionalización & 10,7 & & & 10,8 & 16,2 \\
Recursos Humanos & 13,1 & 16,6 & 18,2 & 13,5 & 14,5 \\
Control y Probidad & 8,3 & 19,0 & 22,7 & 16,2 & 14,0 \\
Proyectos Innovación & 10,7 & 12,0 & 13,6 & 18,9 & 13,0 \\
Atención al Cliente & 5,9 & 2,4 & 9,0 & 10,8 & 6,5 \\
Dirección & 5,9 & & 4,5 & & 3,2 \\
Sistemas de Información & 2,4 & 4,8 & & 5,4 & 3,2 \\
Organización & 4,7 & & & & 3,4 \\
Descentralización & & & & & \\
y Regionalización & 4,7 & 28,5 & 22,7 & & 1,6 \\
Informática & 2,4 & 2,4 & & & \\
Presupuesto y Finanzas & 1,2 & 2,4 & & & \\
Total & & & & & \\
\hline
\end{tabular}

Fuente: Elaboración Propia.

de Rediseño de Procesos, Manuales de Funciones y Procedimientos. Los PFT en Recursos Humanos incluyen aspectos tales como Capacitación, Clima Organizacional, Remuneraciones, Evaluación de Desempeño, Cargos y Funciones.

En las sedes más alejadas de la capital una característica común es que los trabajos se concentran mayoritariamente en dos o tres áreas. En Puerto Montt el $66,1 \%$ de los PFT corresponden a Descentralización y Regionalización, Recursos Humanos y Control y Probidad. En la Serena el $63,6 \%$ se concentra en PFT en las mismas áreas.

Los PFT dirigidos a Estudios se concentran mayoritariamente en Santiago. La centralización que exhiben los servicios públicos en Chile, podría explicar un mayor acceso de los estudiantes a la información.
Debido a la cercanía a las unidades de análisis de los servicios públicos Santiago también representa la sede donde se presenta la mayor amplitud de temas.

Como contrapartida en las sedes de Puerto Montt y La Serena adquieren mayor relevancia los PFT referidos a los procesos de Operacionalización y Racionalización. Mientras en estas sedes ésta área representa el $28,5 \%$ y $22,7 \%$ de los PFT respectivamente, en Santiago y Valparaíso sólo representan el $10 \%$.

\section{2 Áreas de Formación del Programa de Estudio}

La elección del tema de trabajo de los PFT responde a diversos factores que van desde las preferencias de los estudiantes, las exigencias del servicio, que por lo general coincide con ser su lugar 
habitual de trabajo y con las orientaciones del profesor guía. De acuerdo a lo anterior, los énfasis temáticos tienen correspondencia con las áreas incorporadas en el Programa de Estudio.

Las áreas más recurrentes en los PFT de los estudiantes son tres: Administración de Personal, Racionalización Administrativa y Control de la Gestión. Estos cursos, como base analítica de los PFT, están presentes en $60,5 \%$ en los trabajo (Tabla 2). Lo anterior puede explicarse en la medida que los contenidos temáticos de estos ramos se relacionan directamente con las necesidades de los servicios. Del mismo modo representan cursos instrumentales que proporcionan técnicas de gestión, fundamentales para la adecuación y rediseño de organizaciones complejas. Los cursos con un nivel más teórico, aparecen menos presentes en los PFT. Así, los cursos que se inscriben en un a dimensión más conceptual sólo aparecen como elementos básicos de trabajo del $15,8 \%$ de los PFT (Tabla 2).

\section{3 Áreas de Gestión Pública}

Los PFT fueron relacionados con las áreas de interés para la innovación en la gestión pública del Proyecto de Reforma y Estado. Están áreas están explicitadas y permiten comparar la simetría entre los esfuerzos del Estado expresados en éstas áreas y el Interés de los funcionarios del Estado adscritos a un programas de formación profesional. Las áreas del proyecto son: a) Recursos humanos: nuevo trato Estado-funcionarios, dirección pública, clima organizacional.

b) Calidad del servicio: reducción y expedición de trámites, derechos de los usuarios, información oportuna y accesible a los ciudadanos, capacidad de respuesta y solución de reclamos de los ciudadanos, incentivos al buen desempeño.

c) Gestión de servicios: sistematización de un paradigma de la gestión pública, presupuestación por resultados, perfeccionamiento de servicios, comparabilidad y replicabilidad.

d) Participación ciudadana en la gestión: Ley de bases para la participación, suscripción de cartas genéricas de derechos del usuario defensor del ciudadano y del consejo de Diálogo Social.

e) Auditoria, transparencia y probidad en la gestión Pública: reglamentos que implementarán las modificaciones introducidas en la ley $N^{\circ} 18.575$ por la ley de probidad, desarrollo de mecanismos de regulación de libre acceso a la información, fortalecimiento de la auditoria interna del Gobierno.

f) Tecnologías de información y comunicación: Infraestructura para un Estado electrónico, uso de las tecnologías de información en proceso de gestión del Estado.

Los PFT poseen una alta relación con las áreas de gestión pública sugeridas como iniciativas del proceso de modernización del Estado en Chile. El 59,4\% 


\section{Tabla 2}

Universidad de los Lagos

Programa de Formación profesional para el sector Público
Proyectos Finales de Titulación por Áreas de Formación

\begin{tabular}{lcc}
\hline Área de Formación & $\begin{array}{c}\text { Proyectos Finales } \\
\text { de Titulación }\end{array}$ & Porcentaje \\
\hline Administración de Personal & 10 & 26,3 \\
Racionalización Administrativa & 7 & 18,4 \\
Control de Gestión & 6 & 15,8 \\
Teoría administrativa & 4 & 10,5 \\
Dirección Administrativa y Teoría & & \\
de la Decisión & 3 & 7,9 \\
Teoría Organizacional & 3 & 7,9 \\
Planificación Estratégica & 2 & 5,2 \\
Sistema de Inversión Pública & 1 & 2,6 \\
Administración Pública & 1 & 2,6 \\
Métodos y Técnica. Investigación & 1 & 2,6 \\
Total & & \\
\hline
\end{tabular}

Fuente: Elaboración Propia.

de los PFT están relacionados con estas áreas ${ }^{11}$. Ello muestra un alto interés en la materia, en cuanto a iniciativas de modernización y desarrollo de líneas de acción. No se observan diferencias significativas en la selección de trabajos orientados a las áreas de gestión pública entre las sedes estudiadas, en las que se observa un rango entre $54 \%$ y $63 \%$ (Tabla 3 ). Sin embargo, una mayor orientación de los estudiantes hacia trabajos en áreas de gestión pública correspondió a la sede de Santiago, lo que podría ser explicado por la concentración de información en los servicios públicos, coherente con el modelo centralizado, en el que la promoción de las iniciativas de modernización es realizada en primer término en la capital. Con todo, el interés de los estudiantes de regiones (La Serena, Valparaíso y Puerto Montt) sigue siendo muy elevado, al escoger dentro de una variedad extensa de temas en el área de la administración pública.

Un análisis más específico, informa que los alumnos se inclinaron, dentro de las áreas de gestión pública, preferentemente por Gestión de Servicios. Casi la mitad de los trabajos revisados se orientó a esta área (Tabla 4). En un segundo nivel de preferencias se encuentran los trabajos dirigidos a las áreas de Recursos Humanos, Calidad de Servicios y Tecnologías de Información y Comunicación.

11 Este porcentaje corresponde al universo de 185 trabajos analizados. 


\section{Tabla 3}

Universidad de los Lagos

\section{Programa de Formación Profesional para el sector Público Proyectos Finales de Titulación Relacionados con el Proceso de Modernización por Sede}

\begin{tabular}{lccc}
\hline Ciudad / Sede & $\begin{array}{c}\text { Trabajos Finales } \\
\left(\mathbf{N}^{\circ}\right)\end{array}$ & $\begin{array}{c}\text { Relacionados con el } \\
\text { proceso de } \\
\text { modernización }\left(\mathbf{N}^{\circ}\right)\end{array}$ & $\begin{array}{c}\text { Relacionados con el } \\
\text { proceso de } \\
\text { modernización (\%) }\end{array}$ \\
\hline La Serena & 22 & 13 & 59,1 \\
Santiago & 84 & 53 & 63.1 \\
Valparaíso & 37 & 21 & 56,7 \\
Puerto Montt & 42 & 23 & 54,7 \\
Total & 185 & 110 & 59,4 \\
\hline
\end{tabular}

Fuente: Elaboración Propia.

\section{Tabla 4}

Universidad de los Lagos

\section{Programa de Formación Profesional Para el Sector Público}

\section{Proyectos Finales de Titulación por Áreas de Modernización y Sede}

\begin{tabular}{lccccc}
$\begin{array}{l}\text { Iniciativa para la modernización } \\
\text { de la Gestión Pública }\end{array}$ & La Serena & Santiago & Valparaíso & $\begin{array}{c}\text { Puerto } \\
\text { Montt }\end{array}$ & $\%$ \\
\hline Recursos Humanos & 1 & 2 & 1 & 3 & 20.7 \\
Calidad de Servicio & 1 & 3 & 1 & 0 & 17.2 \\
Gestión de Servicios & 1 & 5 & 3 & 4 & 44.9 \\
Participación Ciudadana en la Gestión & 1 & & & & \\
Auditoria, Transparencia y probidad & & & & & 3.4 \\
en la gestión Pública & 1 & & & & \\
Tecnologías de información & & & 3 & 1 & 13.8 \\
y Comunicación & 0 & 0 & 8 & 8 & 100 \\
Total & 5 & 10 & &
\end{tabular}

Fuente: Elaboración Propia.

Sólo un $3.4 \%$, de los PFT se orientó a Auditoria, Transparencia y Probidad en la Gestión Pública. No se encontraron PFT en el área de Participación Ciudadana, lo que podría ser explicado por la reciente incorporación de este tópico en la agenda gubernamental para la administración pública chilena $^{12}$.

En cuanto a las áreas de mayor interés, Gestión de Servicios se encuentra similarmente distribuida entre las sedes analizadas. Se observa también una mayor disposición a algunas áreas por sedes: Recursos Humanos en la sede de Puerto Montt, Calidad de Servicios en la sede de Santiago y Tecnologías de Infor-

12 Recién en el año 2001 a través de un convenio y financiamiento compartido entre el Gobierno de Chile y el Banco Mundial se están trabajando proyectos de fortalecimiento de la sociedad civil y participación ciudadana en la implementación de políticas públicas y la gestión pública. 
mación y Comunicación en la sede de Valparaíso. Estas preferencias específicas podrían ser explicadas por el papel relevante que juegan los profesores tutores en la selección de los trabajos finales.

\section{Conclusiones}

- Las sociedades en desarrollo valoran de manera creciente el aumento del capital humano como una estrategia probada y viable para acercarse al desarrollo. En él, ocupa un lugar central el aumento de las coberturas y calidad de los sistemas de formación profesional. Sin embargo, este convencimiento choca con la falta de recursos y el empleo de estrategias tradicionales que, si bien han aumentado las tasas de escolarización superior, no han corregido la distancia relativa que separa a los países desarrollados y en desarrollo en el nivel educacional de su población.

- La formación profesional de trabajadores, orientada a nuevos segmentos de la población, tradicionalmente al margen de las instituciones de formación, aparece como una alternativa relevante para el mejoramiento del capital humano de las naciones. Programas de formación profesional de trabajadores, como la educación continua y la formación para toda la vida, son cada vez más difundidas en Europa y Estados Unidos. También se observa una creciente incorporación de trabajadores en programas tradicionales de formación profesional de las universidades. Estas tendencias son comprensibles por la alta valoración que la educación ocupa en la so- ciedad, las transformaciones sociales y tecnológicas ocurridas en las últimas tres décadas y los cambios de paradigmas sobre la formación profesional.

- La necesidad de mejorar el capital humano del sector público en Chile, se ha visto reflejada por los nuevos requerimientos que generan los procesos de reforma y modernización del Estado. Contar con recursos humanos calificados, con nuevas capacidades y habilidades para enfrentar las transformaciones sociales y económicas es una condición para el desarrollo y el éxito de estas reformas.

- Los programas de formación profesional y técnica para la administración pública de esta Universidad, han sido concebidos como programas que buscan desarrollar capacidades profesionales para formular, desarrollar, implementar y ejecutar políticas, planes, programas y proyectos públicos, y desempeñarse en posiciones ejecutivas y técnicas de la administración pública. Estudios realizados para evaluar la percepción de los funcionarios públicos sobre estos programas, expresan una alta valoración por las competencias desarrolladas $y$ un alto sentido de pertenencia con sus trabajos, por lo que sería previsible un impacto positivo sobre las instituciones donde trabajan.

- Los programas de formación profesional para trabajadores de la administración pública han resuelto favorablemente la articulación entre formación profesional y experiencia laboral. Dilema que sigue estando presente en los programas de formación 
para la administración pública de jóvenes sin experiencia laboral. Sin embargo, sería sugerible profundizar líneas de investigación sobre los procesos pedagógicos presentes en esta alternativa de formación profesional, que sustenta el aprendizaje en la experiencia de los trabajadores, con importantes efectos sobre las competencias a desarrollar y las me todologías de aprendizaje

- Del estudio de los proyectos finales de titulación en funcionarios públicos adscritos a programas de formación profesionales de trabajadores en $\mathrm{Chi}$ le, se concluye que existe una alta relación entre el Programa de Estudios, sus intereses profesionales y los esfuerzos que el Estado realiza en su proceso modernizador. Los funcionarios desarrollaron iniciativas que se inscriben claramente en las áreas que el Estado considera prioritarios para el mejoramiento de la gestión pública. En funcionarios públicos en proceso de adopción de competencias profesionales, parece suficientemente socializado y compartido el sentido del proyecto modernizador.

\section{Bibliografía Citada}

Banco Mundial (1993), El Milagro de Asia Oriental: El crecimiento económico y las políticas oficiales. Washington D. C.

Banco Mundial (2000), La Educación Superior en los países en Desarrollo. Corporación de Promoción Universitaria. Santiago de Chile.

Bachtemann, Chietoph y Sodoff, Dana (1998), Formación Profesional. CEDEFOP, Revista Europea №13.
Brunner, Jose Joaquin (2000), Innovación en las políticas y políticas de innovación. En: Política de educación superior: ¿tiempo de innovar? Consejo Superior de Educación, Santiago, pp. 30-35.

De Moura, Claudio (2000), Políticas de educación superior: una perspectiva latinoamericana. La educación superior en América Latina y el Caribe. Consejo Superior de Educación, Santiago.

Díaz, Nelson (1996), El programa de forma ción técnica y profesional para funcionarios de la administración del Estado: una experiencia transferible de desarrollo de recursos humanos en Chile. Primer congreso CLAD, Río de Janeiro.

CINTERFOR (1996), Formación y trabajo de ayer para mañana. Papeles de la Oficina $\mathrm{N}^{\circ} 1$. Montevideo.

CINTERFOR (1998), Formación, trabajo y conocimiento. Papeles de la oficina No7.

Dellors, Jacques (1996), La educación encierra un tesoro. Informe a la UNESCO de la Comisión Internacional sobre la educación para el siglo XXI.

Fundación CONFEMETAL (1999), El Plan de Formación de la empresa. Guía práctica para la elaboración y desarrollo. Madrid.

Goetz, J. P. y LeCompte, M. D. (1988), Etnografía y diseño cualitativo de investigación educativa, MORATA, Madrid.

Guerrero, Omar (1992), Políticas y criterios técnicos para la modernización curricular de la formación de gerentes públicos en Iberoamérica. Red lberoamericana de Instituciones de Formación e Investigación en Gerencia Pública. Serie Documentos Técnicos №3. México. 
Leibowics, Julieta (2000), Ante el imperativo de aprendizaje permanente, estrategias de formación continua. CINTERFOR. Papeles de la Oficina $\mathrm{N}^{\circ} 9$.

Mancilla, Marcelo (1984), América Latina: Impacto de la crisis de la década de 1980 y la Administración Pública. Mimeo. Chile. Instituto Profesional de Osorno.

Martínez, Eduardo (1999), Experiencias de alianzas estratégicas Estado-empresas, CINTERFOR, 1999.

Ospina, Sonia (1998), Educación e identidad en la Administración Pública: Dilemas en la construcción de una comunidad para formar profesionales de lo público. Anales del CLAD.www.clad.org.ve/anales2/ ospina.htm

Rivera, Claudio y Neira, Marcelo (2001), Formación profesional de trabajado- res: contextos y tendencias. Programa de Especialización Profesional, Universidad de Los Lagos, Santiago, 2001.

Scholl, E. (1995), Lerning to love the swamp: resaphing education for public service. Journal of Policy Analisis and Management Vol. 14, N², pp. 202220. Estados Unidos.

UNESCO (1997), Políticas de Educación Superior.

Universidad de los Lagos (1994), Decreto Interno número 70 . Chile.

Universidad de los Lagos (1995), Decreto Interno número 934. Chile.

Universidad de los Lagos (2000), Red de Servicios Universitarios Regionales. Chile.

Vial, Joaquín (1996), Ministerio de Economía, Mimeo. Santiago de Chile. 